\title{
Extensions of measures and abstract stochastic processes
}

\section{by}

\author{
E. O. Elliott (Holmdel, N. J.)
}

1. Introduction. In the general theory of stochastic processes the extension of probability measure from finite dimensional distributions to denumerable dimensions is easily effected in most cases. For abstract processes, the theory of compact systems of sets due to Marczewski and Ryll-Nardzewski may be invoked to achieve the extension to denumerable dimensions without the reliance upon topological structures. In the theory of processes with mutually independent random rariables (in the continuous parameter case), the measurability of erents defined by a condition on a nondenumerable collection of random variables has been established. Using a general extension theorem which embodies the essence of this second result and the technique used in obtaining a separable extension of a stochastic process, the (transcountable) extension problem is completed here for abstract processes in a fashion that assures the measurability of events specified by nondenumerable collections of random rariables. This provides a generalization of the notion of separability which is applicable to both abstract and real stochastic processes.

We consider an $(\Omega)$ space $S$ of function space type with an abstract parameter index set $I$ such that the functions $x \in S$ assume values in the abstract spaces $S_{t}, t \in I$, i.e.,

$$
S=\left\{x: x \text { is a function on } I \text { and } x(t) \in S_{t} \text {, for each } t \in I\right\} .
$$

To each space $S_{t}, t \in I$, is associated a $\sigma$-field of subsets $F_{t}$ and we define the families of subsets $R_{J}, J \subset I$, as follows:

$$
\boldsymbol{R}_{J}=\left\{B: B=\left\{x \in S: x(t) \in \beta_{t}, t \in J\right\}, \beta_{t} \in F_{t}, t \in J\right\} .
$$

Special cases of these are the sigma-field $\boldsymbol{F}_{t}=\boldsymbol{R}_{\{t\}}$ and $\boldsymbol{R}=\boldsymbol{R}_{I}$. We assume $S_{t} \in F_{t}$ for each $t \in I$ so that $S \in \boldsymbol{F}_{t}$ also.

Digressing for a moment, let us make the following set and measuretheoretic notational agreements.

(i) $H_{\text {fin }}=\{K: K \subset H, K$ is finite $\}$, 
(ii) $H_{\mathrm{cbl}}=\{K: K \subset H, K$ is countable $\}$,

(iii) $\mu=g[\boldsymbol{H}]$ is the function on the subsets of $\boldsymbol{S}$ such that if $A \subset \boldsymbol{S}$ then $\mu(A)=\operatorname{Inf}\left\{\sum_{B \in G} g(B): A \subset \bigcup G, G \in \boldsymbol{H}_{\mathrm{cbl}}\right\}$,

(iv) $\mu=\varphi[\bmod N]$ is the function on the subsets of $S$ such that if $A \subset S$ then $\mu(A)=\operatorname{Inf}\{\varphi(A-N): N \in N\}$.

(v) $\mu / \boldsymbol{H}$ is the function $\mu$ with its domain restricted to (members of the family) $\boldsymbol{H}$.

(vi) $\Sigma(\boldsymbol{G})=$ the smallest sigma-field containing $\boldsymbol{G}=\bigcap\{\boldsymbol{H}: \boldsymbol{G} \subset \boldsymbol{H}$, $\boldsymbol{H}$ is a $\sigma$-field .

The following additional families of subsets of $S$ may now be defined. Let $\boldsymbol{A}_{J}^{*}=\Sigma\left(\boldsymbol{R}_{J}\right)$ and $\boldsymbol{A}^{*}=\bigcup\left\{\boldsymbol{A}_{J}^{*}: J \in I_{\mathrm{cbl}}\right\}$ and assume that $\boldsymbol{F}_{J}, J \in I_{\mathrm{fin}}$ is a sigma-field containing $\boldsymbol{A}_{j}^{*}$ determined by restrictions of the $x$ 's values on the parameter set $J .\left(\boldsymbol{F}_{J} \subset \boldsymbol{F}_{J^{\prime}}\right.$ when $\left.J \subset J^{\prime} \in I_{\text {fin }}\right)$.

We assume a measure $\tau^{*}$ on the sigma-field $\boldsymbol{F}^{*}=\bigcup\left\{\boldsymbol{F}_{J}^{*}: J \in I_{\mathrm{cbl}}\right\}$ where $\boldsymbol{F}_{J}^{*}=\Sigma\left(\bigcup\left\{\boldsymbol{F}_{J^{\prime}}: J^{\prime} \in J_{\text {fin }}\right\}\right)$ is given (or has been obtained from measures on $\boldsymbol{F}_{J^{\prime}}$ by extension based on compact systems of sets ([1], [2])). Our goal is to modify $\tau^{*}$ by extending it to a universal sigma-field $\boldsymbol{F}$ of measurable subsets of $\boldsymbol{S}$ which includes $\boldsymbol{R}$ and $\boldsymbol{F}^{*}$. Thus, the members of these two families are measurable under the resultant measure so when $I$ is nondenumerable, certain events specified by a nondenumerable collection of random variables are measurable. This goal is accomplished with a generalization of an extension theorem [3] which has its inception in the theory of mutually independent processes with a continuous parameter [4]. A device in this application is familiar for the obtaining of a separable standard extension of a stochastic process [5]. As in [4] there is no need to restrict consideration to finite (or probability) measures or $\sigma$-finite measures, but we do not undertake such complete generality at this time. Thus, we do not label $\tau^{*}$ as a probability measure here (for possible future reference) but the reader may think of it as such since $\tau^{*}(\boldsymbol{S})<\infty$ is often required.

As one final notational device, we let $A_{J}$ denote

$$
\{x \in S: \text { for some } y \in A, x(t)=y(t) \text { for each } t \in J\} \text {. }
$$

and write $A_{t}=A_{\{l\}}$. Thus, when $A \in R, A=A_{J} \cap A_{I-J}$ for any $J \subset I$, . and $A=\bigcap\left\{A_{t}: t \in I\right\}$. Now, if $A$ is any subset of $S$ and $J \subset I$ then $A_{J}$ is the cylinder in $S$ over the projection of $A$ onto the subspace $\prod\left\{S_{t}: t \in J\right\}$, the cartesian product of the spaces $S_{t}, t \in J$. (For a set-theoretic method of handling the mechanics of product spaces some readers might be interested in $([4], \S 5)$.)

Throughout this paper we assume $I$ is nondenumerable.
2. The extension of $\tau^{*}$. We let $\bar{\tau}=\tau^{*}\left[\boldsymbol{F}^{*}\right]$ be the outer measure extension of $\tau^{*}$ on $S$ so that

$$
\bar{\tau}(A)=\operatorname{Inf}\left\{\tau^{*}(B): A \subset B \in \boldsymbol{F}^{*}\right\},
$$

whenever $A \subset S$, and specify the further modification of $\tau^{*}$ by means of the following:

Definitions.

(1) $\boldsymbol{B}=\left\{A \cap B: A \in F_{J}^{*}, B \in \boldsymbol{R}_{I-J}, J \in I_{\mathrm{cbl}}\right\}$,

(2) $C=\left\{\alpha: \alpha=\bigcup\{a(t): t \in I\}, a(t) \in \boldsymbol{F}_{t}, t \in I\right\}$.

If $\alpha \in C$ we take the notational liberty of writing $\alpha=\bigcup\left\{\alpha_{t}: t \in I\right\}$ where now $\alpha_{t}(=a(t))$ has a new, but reserved, meaning.

(3) $N(A)=\left\{a \in C: A \in F^{*}, \bar{\tau}\left(A \alpha_{t}\right)=0, t \in I\right\}$,

(4) $N^{*}(A)=\left\{D: A=\bigcup\left\{A^{k}: k=1,2, \ldots\right\}\right.$,

$$
\begin{aligned}
& \left.D=\bigcup\left\{A^{k} \alpha^{k}: k=1,2, \ldots\right\}, a^{k} \in N\left(A^{k}\right), k=1,2, \ldots\right\}, \\
& \boldsymbol{N}=\bigcup\left\{\boldsymbol{N}^{*}(A): A \in \boldsymbol{F}^{*}\right\}, \\
& \boldsymbol{v}=\bar{\tau}[\bmod \boldsymbol{N}], \\
& \boldsymbol{F}=\{A \subset \boldsymbol{S}: A \text { is a } v \text {-measurable set }\} .
\end{aligned}
$$

The family $N$ in (5) above is our generalization of the nilsets in [3]. We begin with an intuitive but important orientational

2.1. Theoreni. If $\mathcal{A} \subset S$ then

$$
\bar{\tau}(A)=\operatorname{Liminf}_{\Sigma \in I_{\mathrm{cbl}}}\left\{\bar{\mu}\left(A_{K}\right): \mu=\bar{\tau} / F_{K}^{*}\right\}=\operatorname{Liminf}_{K \in I_{\mathrm{cbl}}} \operatorname{Inf}\left\{\bar{\tau}\left(A^{\prime}\right): A \subset A^{\prime} \epsilon F_{K}^{*}\right\} .
$$

Proof. Recall that $A_{E}$ is the cylinder in $S$ over the projection of $A$ onto the space $\prod\left\{S_{t}: t \in K\right\}$. Observe that for some $J \in I_{\mathrm{cbl}}$ we have that both

and

$$
\bar{\tau}(A)=\bar{\tau}\left(A^{\prime}\right), \quad A \subset A^{\prime} \in F_{J}^{*}
$$

$$
\operatorname{Liminf}_{K \in I_{\mathrm{cbl}}}\left\{\bar{\mu}\left(A_{K}\right): \mu=\bar{\tau} / \boldsymbol{F}_{K}^{*}\right\}=\bar{\mu}\left(A_{J}\right)
$$

are satisfied $\left(\mu=\bar{\tau} / F_{J}^{*}\right)$.

Since $A \subset A^{\prime} \in F_{J}^{*}$ we have $A_{J} \subset A^{\prime}$ and hence $\bar{\tau}(A)=\bar{\tau}\left(A_{J}\right)=\bar{\tau}\left(A^{\prime}\right)$ since $A \subset A_{J}$. Since for some $A^{\prime \prime} \in F_{J}^{*}, A_{J} \subset A^{\prime \prime}$ and $\bar{\mu}\left(A_{J}\right)=\bar{\tau}\left(A^{\prime \prime}\right)$ it follows that $\bar{\tau}\left(A^{\prime \prime}\right)=\bar{\tau}\left(A^{\prime} A^{\prime \prime}\right)=\bar{\tau}\left(A^{\prime}\right)=\bar{\tau}\left(A_{J}\right)$ completing the proof.

Fundamental to our goal is the next

2.2. THEOREMr. If $A \in \boldsymbol{F}^{*}$ and $D \in N^{*}(A)$ then $\bar{\tau}(A-D)=\bar{\tau}(A)$. 
Proof. Let. $A=\bigcup\left\{A^{k}\right\}, \quad D=\bigcup\left\{A^{k} \alpha^{k}\right\}$ where $a^{k} \in N\left(A^{k}\right)$, $k=1,2, \ldots$, and set $\beta_{t}=\bigcup\left\{A^{k} \alpha_{t}^{k}\right\}, t \in I$, and let $B=A-D$. Then

$$
D=\bigcup\left\{\beta_{t}: t \in I\right\} \quad \text { and } \quad B=A-\bigcup_{t \in I} \beta_{t}=\bigcap_{t \in I}\left(A-\beta_{t}\right) .
$$

Now suppose $J \in I_{\mathrm{cbl}}$ is the smallest such for which $A \in F_{J}^{*}$. Then, whenever $J \subset J^{\prime} \in I_{\mathrm{cbl}}$, we have $B_{J^{\prime}}=\bigcap_{t \in J^{\prime}}\left(A-\beta_{t}\right) \in \boldsymbol{F}_{J^{\prime}}^{*}$. Using the previous theorem we infer

$$
\begin{aligned}
\bar{\tau}(B) & =\operatorname{Liminf}_{\bar{K} \in I_{\mathrm{cb1}}}\left\{\bar{\mu}\left(B_{K}\right): \mu=\bar{\tau} / \boldsymbol{F}_{K}^{*}\right\}=\operatorname{Sup}_{K} \operatorname{Inf}\left\{\bar{\mu}\left(B_{J^{\prime}}\right): \mu=\bar{\tau} / \boldsymbol{F}_{J^{\prime}}^{*}\right\} \\
& \geqslant \operatorname{Sup}_{K} \operatorname{Inf}_{J^{\prime} \supset K} \bar{\tau}\left(B_{J^{\prime}}\right),
\end{aligned}
$$

and consequently for some $J^{\prime} \in I_{\mathrm{cbl}}, J \subset J^{\prime}$, the right-hand side of the inequality is equal

$$
\bar{\tau}\left(B_{J^{\prime}}\right)=\bar{\tau}\left(\bigcap_{t \in J^{\prime}}\left(A-\beta_{t}\right)\right)=\bar{\tau}\left(A-\bigcup_{t \in J^{\prime}} \beta_{t}\right)=\bar{\tau}(A)-\bar{\tau}\left(\bigcup_{t \in J^{\prime}} \beta_{t}\right)=\bar{\tau}(A)-0
$$

and we conclude $\bar{\tau}(B) \geqslant \bar{\tau}(A) \geqslant \bar{\tau}(B)$ to complete the proof.

2.3. Theorem. If $B \in F^{*}, \bar{\tau}(B)<\infty$ and $D \in N$ then $\bar{\tau}(B-D)=\bar{\tau}(B)$.

Proof. Suppose $A \in F^{*}$ and $D \in N^{*}(A)$ then, $D \subset A$ and since $A$ is $\bar{\tau}$ measurable we have

$$
\begin{aligned}
\bar{\tau}(B-D) & =\bar{\tau}(B-D-A)+\bar{\tau}(A(B-D))=\bar{\tau}(B-A)+\bar{\tau}(A B-D) \\
& =\bar{\tau}(B)-\bar{\tau}(A B)+\bar{\tau}(A B-D)
\end{aligned}
$$

and checking that $D \in N^{*}(A B)$ we infer from the previous theorem that $\bar{\tau}(A B-D)=\bar{\tau}(A B)$ and then conclude $\bar{\tau}(B-D)=\bar{\tau}(B)$.

2.4. THEOREM. The set function $v$ is an outer measure on $S, v(A)$ $=\bar{\tau}(A)$ and $A$ is $\nu$ measurable whenever $A \in F^{*}$, and $\nu(D)=0$ whenever $D \in N$, and $v=\nu\left[F^{*} \cup N\right]$.

Proof. Noting that $\bar{\tau}=\bar{\tau}\left[F^{*}\right]$ the conclusion is an immediate consequence of the fact that $\boldsymbol{N}$ is closed to countable unions which is easily checked. This is well known [4] but for completeness we sketch a proof. First, to check that $v$ is an outer measure we must verify that $v(A)$ $\leqslant \sum_{k=1}^{\infty} v\left(B^{k}\right)$ whenever $A \subset S, A \subset \bigcup\left\{B^{k}: k=1,2, \ldots\right\}, B^{k} \subset S, k=1,2, \ldots$ Since $\nu(C)=\operatorname{Inf}\{\bar{\tau}(C-D): D \in \boldsymbol{N}\}$ for each $O \subset S$, we may use the fact that $N$ is closed to countable unions to secure $D^{k} \in \mathbf{N}, k=0,1, \ldots$, so that

$$
\nu(A)=\bar{\tau}\left(A-D^{0}\right) \quad \text { and } \quad v\left(B^{k}\right)=\bar{\tau}\left(B^{k}-D^{k}\right), \quad k=1,2, \ldots
$$

Then letting $D=\bigcup\left\{D^{k}: k=0,1, \ldots\right\}$ we see that $D \in N$ and $D^{k} \subset D$ and hence $\nu(A)=\bar{\tau}(A-D)$ and $\nu\left(B^{k}\right)=\bar{\tau}\left(B^{k}-D\right), k=1,2, \ldots$ Now, $A-D \subset \bigcup\left\{B^{k}-D: k=1,2, \ldots\right\}$ and since $\bar{\tau}$ is an outer measure,

$$
\bar{\tau}(A-D) \leqslant \sum_{k=1}^{\infty} \bar{\tau}\left(B^{k}-D\right)
$$

and the desired conclusion follows.

Next, to check that $\nu=v\left[F^{*} \cup N\right]$ we suppose $A \subset S$ and let $D \in N$ be such that $\nu(A)=\bar{\tau}(A-D)$. Then, using the fact that $\bar{\tau}=\bar{\tau}\left[F^{*}\right]$ we suppose $r>0$ and $\bar{\tau}(A-D)+r \geqslant \sum_{k=1}^{\infty} \bar{\tau}\left(B^{k}\right)$ where $B^{k} \in F^{*}$ and $A-D$ $\subset \bigcup_{k=1}^{\infty} B^{k}$. Then, since $\nu\left(B^{k}\right)=\bar{\tau}\left(B^{k}\right)$ we conclude $\nu(A)+r \geqslant \sum_{k=1}^{\infty} \nu\left(B^{k}\right)$. Hence, letting $B^{0}=D$ we see that $A \subset \bigcup\left\{B^{k}: k=0,1, \ldots\right\}$ and as $0 \leqslant v\left(B^{0}\right) \leqslant \bar{\tau}\left(B^{0}-D\right)=\bar{\tau}(\varnothing)=0, \quad v\left(B^{0}\right)=0$ and we infer $v(A)+r$ $\geqslant \sum^{\infty} \nu\left(B^{k}\right) \geqslant \nu(A)$, and since $\left\{B^{k}: k=0,1, \ldots\right\} \in\left(\boldsymbol{F}^{*} \cup \boldsymbol{N}\right)_{\text {cbl }}$ we conclude $\boldsymbol{v}=\boldsymbol{v}\left[\boldsymbol{F}^{*} \cup \boldsymbol{N}\right]$. To see that members of $\boldsymbol{F}^{*}$ are $\nu$ measurable it is then only necessary to check that $\nu(A)=\nu(A B)+\nu(A-B)$ whenever $A \in F^{*} \cup N$ and $B \in F^{*}$ which is clear.

Using a familiar technique from the theory of separable processes ([5], Lemma 2.1) we obtain another fundamental

2.5. TheoreM. If $B \in \boldsymbol{R}_{I-J^{\prime}}, J^{\prime} \in I_{\mathrm{cbl}}, \bar{\tau}(\boldsymbol{S})<\infty, \alpha_{t}=\boldsymbol{S}-B_{t}$ for each $t \in I$, then for some $J \in\left(I-J^{\prime}\right)_{\mathrm{cbl}}$,

$$
\bigcup\left\{\alpha_{t}: t \in I-J\right\} \in \mathbf{N}\left(B_{J}\right) \text {. }
$$

Proof. We proceed by induction and let $t_{1}$ be any member of $I-J^{\prime}$ and suppose that $J_{k}=\left\{t_{1}, \ldots, t_{k}\right\}$ has been defined in accordance with the following scheme: Let

$$
r_{k}=\operatorname{Sup}\left\{\bar{\tau}\left(B_{J_{k}} \alpha_{t}\right): t \in I\right\}
$$

If $r_{k-1}>0$ and $r_{k}=0$ then take $J=J_{k}$. If $r_{k}>0$ then let $t_{k+1}$ be choser in $I-J^{\prime}-J_{k}$ so that

$$
\bar{\tau}\left(B_{J_{k}} a_{t_{k+1}}\right)>r_{k}\left(1-k^{-1}\right) \text {. }
$$

Now, with $J_{k+1}=J_{k} \cup\left\{t_{k+1}\right\}$, we see that $B_{J_{k+1}}=B_{J_{k}} \cap B_{t_{k+1}} \subset B_{J_{k}}$ and hence that $r_{k+1} \leqslant r_{k}$. Now, if $r_{k}>0$ for all $k=1,2, \ldots$, then

$$
G=\left\{B_{J_{k}} a_{t_{k+1}}: k=1,2, \ldots\right\}
$$

is a disjoint subfamily of $\boldsymbol{A}^{*} \subset \boldsymbol{F}^{*}$ and consequently

$$
\bar{\tau}(\bigcup G)=\sum_{k=1}^{\infty} \bar{\tau}\left(B_{J_{k}} \alpha_{t_{k+1}}\right) \leqslant \bar{\tau}(S)<\infty
$$

Fundamenta Mathematicae, T. LXXII 
and hence

Thus

$$
\lim _{k \rightarrow \infty} \bar{\tau}\left(B_{J_{k}} \alpha_{t_{k+1}}\right)=0
$$

$$
\lim _{k \rightarrow \infty} r_{k}\left(1-k^{-1}\right)=\lim _{k \rightarrow \infty} r_{k}=0,
$$

and taking $J=\bigcup\left\{J_{k}\right\}$ we have

so that

$$
\operatorname{Sup}\left\{\tau\left(B_{J} \alpha_{t}\right): t \in I\right\} \leqslant r_{k}
$$

$$
\bar{\tau}\left(B_{J} \alpha_{t}\right)=0 \quad \text { whenever } \quad t \in I-J .
$$

Defining $\beta_{t}=\alpha_{t}, t \in I-J, \beta_{t}=\varnothing, t \in J$, we have $\bigcup\left\{\beta_{t}: t \in I\right\}=\bigcup\left\{\alpha_{t}\right.$ : $t \in I-J\} \in C$ which yields the conclusion $\bigcup\left\{\alpha_{t}: t \in I-J\right\} \in N\left(B_{J}\right)$.

2.6. THEOREM. If $A \in \boldsymbol{B}$ and $\bar{\tau}(\boldsymbol{S})<\infty$ then for some $D \in \boldsymbol{N}$ and $A^{\prime} \in \boldsymbol{F}^{*}, A=A^{\prime}-D$.

Proof. Suppose $A=B^{\prime} \cap B$ where $B^{\prime} \in \boldsymbol{F}_{J^{\prime}}^{*}$ and $B \in \boldsymbol{R}_{I-J^{\prime}}$ and employ the previous theorem to secure $J \in\left(I-J^{\prime}\right)_{\text {cbl }}$ such that $\beta=\bigcup\left\{\beta_{t}\right.$ : $t \in I\} \in \boldsymbol{N}\left(B_{J}\right)$ where $\beta_{t}=S-B_{t}, t \in I-J$, and $\beta_{t}=\varnothing$ for $t \in J$. Then taking $A^{\prime}=B^{\prime} \cap B_{J}$ we have $A^{\prime} \in F^{*}$ and taking $D=A^{\prime} \beta$ we infer from the fact $A^{\prime} \subset B_{J}$ that $\beta \in N\left(A^{\prime}\right)$ and hence $D \in N^{*}\left(A^{\prime}\right)$ so $D \in N$. Clearly, $B_{I-J}=S-\beta$ so $A=B^{\prime} B_{J} B_{I-J}=A^{\prime}(S-\beta)=A^{\prime}-\beta=A^{\prime}-D$ which completes the proof.

2.7. THEOREM. If $B \in \boldsymbol{B}$ and $\bar{\tau}(\boldsymbol{S})<\infty$ then $B$ is $v$ measurable and $\nu(B)=\bar{\tau}(B)$.

Proof. By 2.6 let $B^{\prime} \in \boldsymbol{F}^{*}$ and $D \in \boldsymbol{N}$ be such that $B=B^{\prime}-D$ so that $\bar{\tau}(B)=\bar{\tau}\left(B^{\prime}-D\right)=\bar{\tau}\left(B^{\prime}\right)$. Now $\nu(B)=\bar{\tau}\left(B-D^{\prime}\right)$ for some $D^{\prime} \in N$. Hence $v(B)=\bar{\tau}\left(B^{\prime}-\left(D \cup D^{\prime}\right)\right)$ and since $D \cup D^{\prime} \in N, \bar{\tau}\left(B^{\prime}-\left(D \cup D^{\prime}\right)\right)$ $=\bar{\tau}\left(B^{\prime}\right)$ and we conclude $\nu(B)=\bar{\tau}(B)$.

To see that $B$ is measurable, we need simply note that $B$ is the difference between the two measurable sets $B^{\prime}$ and $D$ and is therefore measurable.

As a consequence of the foregoing theorems, we see that the measure $\tau^{*}$ on the sigma-field $F^{*}$ can be extended to a measure $v / F$ on the sigmafield $\boldsymbol{F}$ which includes both $\boldsymbol{F}^{*}$ and $\boldsymbol{R}$. Furthermore, if $A \in \boldsymbol{R}$ then for some $J \in I_{\text {cbl }} v(A)=v\left(A_{J}\right)$. Thus $\nu$ has properties similar to but considerably more general than those of a probability measure associated with a separable stochastic process of function space type. In this way, separability is generalized not only to an abstract setting, but also is freed of the special topological structure and restrictions required of real stochastic processes. In the next section we look briefly at some variants of this extension which are not quite as general and relate more closely to the classical theory.
3. Alternate extensions. Two alternate extensions will be briefly described. We mention these because they are appropriate in some applications to real processes. In definition (2) of $C$ we may add either of two additional conditions that $a(t)$ must satisfy. The first is that $\tau^{*}(a(t))>0$ or $a(t)=\varnothing$ for each $t \in I$. This change would then be reflected in definitions $(3)$ to $(5)$ and result in a measure $\nu_{1}$ given by (6). Under this measure not all members of $\boldsymbol{R}$ are measurable. Instead those members $B$ of $\boldsymbol{R}$ for which $B_{t}=S$ or $\tau^{*}\left(S-B_{t}\right)>0$ for each $t \in I$ are found to be measurable. (Altering (1) by adding this condition on $B$ provides a means for using the foregoing theorems with the modified definitions to reach this result.)

The second alternate extension is for the case when the spaces $S_{t}$ are topological. Then the condition that $a(t)$ be open in $S_{t}$ for each $t \in I$ may be added to (2). Definition (6) then results, in a similar manner, with a measure $v_{2}$ under which members $B$ of $R$ for which $B_{t}$ is closed for each $t \in I$ are measurable. This second case strongly resen bles that of a classical separable process. The big exception is that the spaces $S_{t}$ may not be related to one another and the sets $B_{t}$ are arbitrary closed sets.

\section{References}

[1] E. Marczewski, On compact measures, Fund. Math. 40 (1953), pp. 113-124.

[2] J. Pfanzagl and W. Pierlo, Compact systems of sets, Lecture Notes in Mathematics 16 (1966), Berlin.

[3] E. 0. Elliott, An extension theorem for obtaining measures on uncountable product spaces, Proc. Amer. Math. Soc. 19 (1968), pp. 1089-1093.

[4] - and A. P. Morse, General product measures, Trans. Amer. Math. Soc. 110 (1964), pp. 245-283.

[5] J. L. Doob, Stochastic processes, New York 1953.

BELL TELEPHONE LABORATORIES, INCORPORATED Holmciel, New Jersey

Reģu par la Rédaction le 10. 3. 1970 